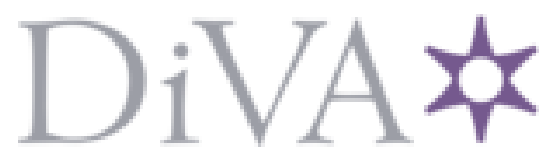

http://www.diva-portal.org

\title{
Postprint
}

This is the accepted version of a paper presented at IEEE PES General Meeting 2016.

Citation for the original published paper:

Singh, R S., Baudette, M., Hooshyar, H., Vanfretti, L., Almas, M S. et al. (2016)

'In Silico' Testing of a Decentralized PMUData-Based Power Systems Mode Estimator.

In: IEEE conference proceedings

N.B. When citing this work, cite the original published paper.

Permanent link to this version:

http://urn.kb.se/resolve?urn=urn:nbn:se:kth:diva- 185010 


\section{'In Silico' Testing of a Decentralized PMU Data-Based Power Systems Mode Estimator}

\author{
Ravi Shankar Singh, Maxime Baudette \\ Hossein Hooshyar, Luigi Vanfretti \\ M. Shoaib Almas \\ KTH Royal Institute of Technology \\ Stockholm, Sweden \\ Email: rssingh@kth.se
}

\author{
Stig Løvlund \\ Luigi Vanfretti \\ Statnett SF \\ Oslo, Norway \\ Email: luigi.vanfretti@ statnett.no
}

\begin{abstract}
This paper presents the software implementation of a Phasor Measurement Unit (PMU) data-based mode estimation application in a decentralized mode estimation architecture. This work builds from previous efforts in the development of a mode estimator implemented using a centralized architecture, meaning a set of modes were estimated for the whole system in a single processing location. One drawback of mode estimators that use centralized mode architecture is that the observability and therefore estimation of important low-damped local electromechanical modes can be affected by the higher observability of other dominant modes of the systems (e.g inter-area modes). This work proposes, implements and tests a decentralized architecture in order to increase the observation capability to provide better estimates of local low damped oscillations. In this architecture, the data from a single PMU or a group of local PMUs could be processed by a processor to estimate the modal parameters observed at a specific part of the grid or observed by specific group of PMUs. The decentralized architecture and results of tests are presented in this paper together with comparison with a centralized architecture.
\end{abstract}

Index Terms-Phasor Measurement Unit (PMU), Mode Estimation, Inter-area Oscillations, Decentrlized Mode Estimation.

\section{INTRODUCTION}

Real-time monitoring has become important for reliable operations of power system. Wide Area Monitoring System (WAMS) is a key element for reliably monitor and control of power systems. WAMS applications provide critical information about system's behaviour, for example, modal properties, oscillation interaction paths, etc. [1]. Modal frequencies and damping are useful indicators of power system stress, which usually increases with increased load or reduced grid capacity. Timely extraction of these and related parameters from system measurements has already demonstrated value for near realtime power system dynamics monitoring and early warning.

Over the past decade, a lot of research has been done to utilize PMU networks and synchrophasor data in WAMS for real-time monitoring applications. In addition, applications utilizing PMU data have been developed and tested for realtime monitoring applications [1], [3] and [4]. These applications function in a centralized architecture where the modeestimator acquires data from all the PMUs and processes all the data to observe the modes present and estimate the modal properties. Although research in [3] indicate that other architectures can aid in the performance of mode estimation applications, no experiments (i.e. laboratory-based with PMUs in the loop) or field implementation have been attempted.

This paper implements a decentralized mode estimator application starting from a centralized estimator presented in [2] to gain location based insight on modal behaviour and achieve a better observability of local, low damped oscillations in the grid. A comparison between both centralized and decentralized architectures is also presented in the context of identifying and estimating local forced oscillations.

The remainder of this paper is organized as follows: Section II describes the original mode-estimator application used for the tests. Section III presents the new decentralized architecture based on the same application. Generation of synthetic PMU data for the tests is described in Section IV. Tests and results are presented in Section V and conclusions are drawn in Section VI.

\section{Real-Time Mode Estimator Software APPLICATION}

The centralized mode estimator application, used in this study, was developed in LabVIEW and exploits PMU measurements to compute mode estimates and characteristic system matrices in real-time. The application is described briefly in this section.

\section{A. Estimation Methods}

The mode estimator application employs measurementbased system identification methods to estimate a power system's modal properties. This approach is not limited by system size or its dynamic behaviour but depends highly on the availability of high quality time-domain signals in realtime [4].

Depending on the system response, continuous measurements received from PMUs may be termed either ambient data or ringdown/transient data [4]. This mode estimator application is able to identify ringdown conditions by detecting high energy oscillations using a method proposed in [7]. For ambient data, this application runs an ARMA-based, Modified 
Yule-Walker method (as described in [4] and [6]) to estimate electromechanical modal parameters. If ringdown condition is detected, the application uses a separate Ringdown Analyzer algorithm which is based on Eigensystem Realization Algorithm (ERA) [4] and operates only for the ringdown portion of the system response.

\section{B. Data Acquisition}

This application is capable of acquiring PMU data using $S^{3}$ DK tool-kit [2], which was built on LabVIEW platform. This tool-kit harnesses real-time PMU signals in the IEEE C37.118.2 standard format and converts the signals into LabVIEW data types. The stream of data from $\mathrm{S}^{3} \mathrm{DK}$ is then used by the subsequent part of the application in LabVIEW. A buffer facilitates asynchronous data transfer from the acquisition part of the application to the pre-processing part [2].

\section{Centralized versus Decentralized ARCHITECTURE}

PMUs can be placed at various locations of the grid to transmit synchrophasor data to a phasor data concentrator (PDC). In a traditional WAMS, there may exist several local PDCs which could be owned by different utilities. These PDCs are connected to a Super PDC in a control center owned by the Transmission System Operators (TSOs). In a centralized architecture, all the data from various points are fed to the Super PDC and then to a software to perform mode estimation and monitoring.

This paper tests a decentralized architecture for mode estimation where the modal characteristics are estimated at a local level instead of centralized control centres. In practise, local level mode estimates could also be sent periodically to the TSO control center for analysis and record of the system's modal properties on a macroscopic level.

For testing purposes, the mode-estimator application was designed to operate in two different architectures:

\section{A. Centralized Mode Estimation}

In this case, the mode estimation algorithm sits centrally and processes the data received from all the PMUs placed throughout the network. It estimates single set of modal parameters for the whole system.

\section{B. Decentralized Mode Estimation}

This architecture allows the application to apply the mode estimations algorithm separately to individual PMUs or groups of PMUs. This means that for each PMU or each group of PMUs, there is a separate processing unit to run the algorithm. The estimated modes by the local processing units could be collected and sent to a higher level. Decentralized mode estimation aims to increase identification capability of oscillations at more local level that may be neglected by centralized architecture.

\section{Synthetic PMU Data Generation}

The application was tested 'in silico' using the system response of a simulated state-space system. The simulations were executed by a LabVIEW module using the normalized system characteristic matrices (A,B,C and D) obtained by linearising the KTH-NORDIC 32 model presented in [8]. In the model, there are 52 buses, 52 transmission lines, 28 transformers and 20 generators (connected to buses 1-20). The network can be divided into two distinct areas and has two critical inter-area oscillatory modes whose parameters are shown in Table I.

TABLE I

CRITICAL MODES OF THE KTH-NORDIC 32 NETWORK

\begin{tabular}{|c|c|c|}
\hline Mode & Frequency & Damping Ratio \\
\hline Mode 1 & $0.499 \mathrm{~Hz}$ & $3.18 \%$ \\
\hline Mode 2 & $0.732 \mathrm{~Hz}$ & $3.52 \%$ \\
\hline
\end{tabular}

Observable paths of the two inter-area modes were already known. According to [8], the dominant inter-area oscillation path for Mode 1 was identified to be 52-51-35-37-38-40-4849-50. Similarly, buses 50-49-44-47 formed the dominant path for Mode 2. These paths were used as a reference for selecting measurement points to derive synthetic synchrophasors from the simulations.

To generate ambient data, the test system was excited using zero mean Gaussian noise ( $\sigma=0.2$ ) representing random lowintensity load variations at each of the 32 load buses. To assess the ringdown algorithm, series of impulses repeating every 5 seconds and of amplitude 500 p.u. were imposed on buses 38 and 42 . These impulses representing recurring faults were used to excite the system. The response of the system (voltage magnitude) in both the above cases was measured using four locations (representing PMUs) which were placed at points along the maximum observability paths (Bus No. 38, 40, 44 and 49) to observe both of the modes. The sampling rate for data recording was 50 samples per second to imitate the sampling rate of a real PMU.

\section{REsults}

This section presents the results calculated using the ambient and transient data recorded as mentioned in Section IV. The measured time series data was fed to the mode estimator and modes were estimated using decentralized and centralized architecture. The estimates obtained were stored and analysed to assess the efficacy of the estimator in finding the desired frequency and damping ratios of the modes. Section V-A presents the results from the decentralized architecture and showcases location specific insights provided by this architecture. An example of centralized estimates is also presented in the section. Section V-B presents the comparison of two architectures for estimating forced local oscillations. During the test, each simulation was run till 6000 estimates were recorded. So, the statistical data calculated for each case are based on a sample space of 6000 estimates. 

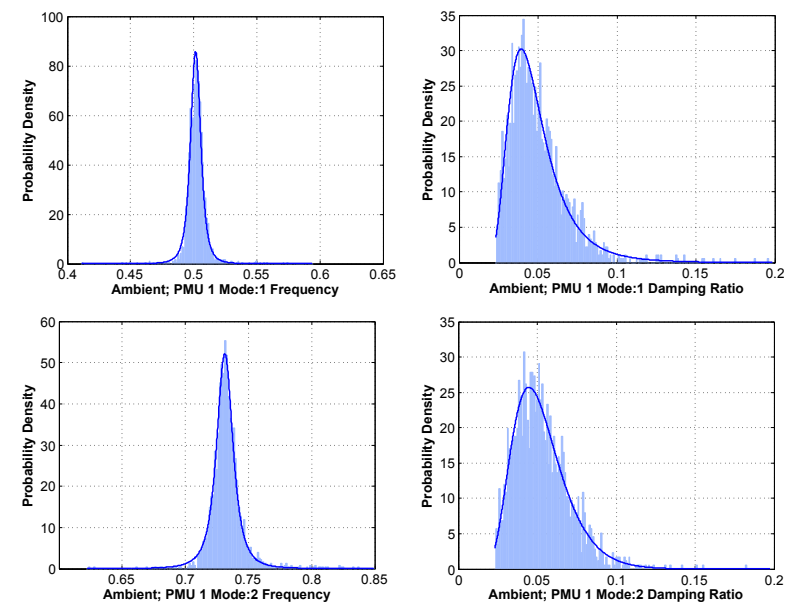

Fig. 1. Estimates form PMU1 data in ambient conditions; TOP: PDF of Mode 1 frequency (left), PDF of Mode 1 damping ratio (right); BOTTOM: PDF of Mode 2 frequency (left), PDF of Mode 2 damping ratio (right).
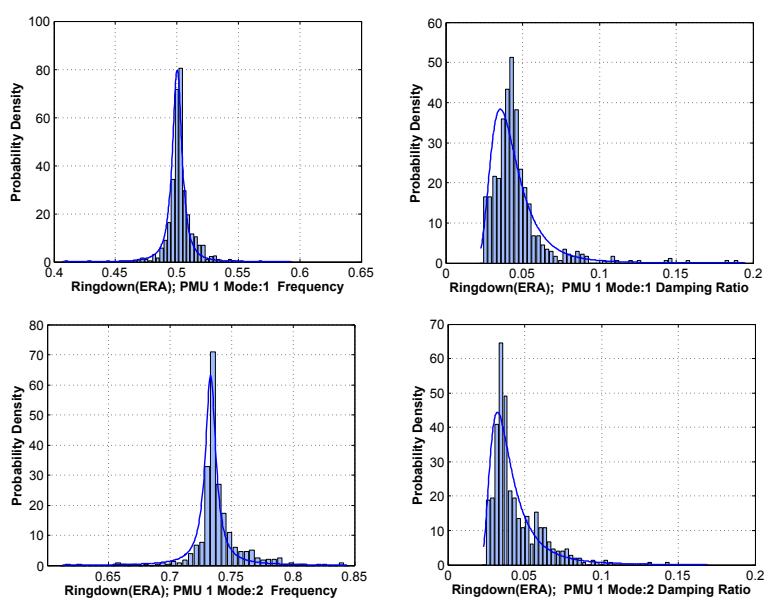

Fig. 3. Estimates form PMU1 data in ringdown conditions; TOP: PDF of Mode 1 frequency (left), PDF of Mode 1 damping ratio (right); BOTTOM: PDF of Mode 2 frequency (left), PDF of Mode 2 damping ratio (right).

\section{A. Decentralized Mode Estimation: Localized Insights on Modal Activities}

1) Ambient Data: In case of ambient data, estimates were calculated on a moving window of 30000 samples of the recorded time series data. Statistics calculated based on the estimates result obtained is presented in Table II. The mean and standard deviation of the frequency and damping of the two modes identified are presented for all the PMUs which were used in the decentralized architecture of mode estimation.

Figs. 1 and 2 display the Probability Distribution Function (PDF) of frequency and damping ratio estimates of the two inter-area modes calculated using data from individual PMUs (PMU 1 at bus 38 and PMU 4 at bus 49) as part of decentralized mode estimation architecture. The decentralized estimation architecture allows to monitor the modes from a more local perspective. As observed from the PDFs of modal frequencies, the activity of Mode 1, observed by PMU 4 at
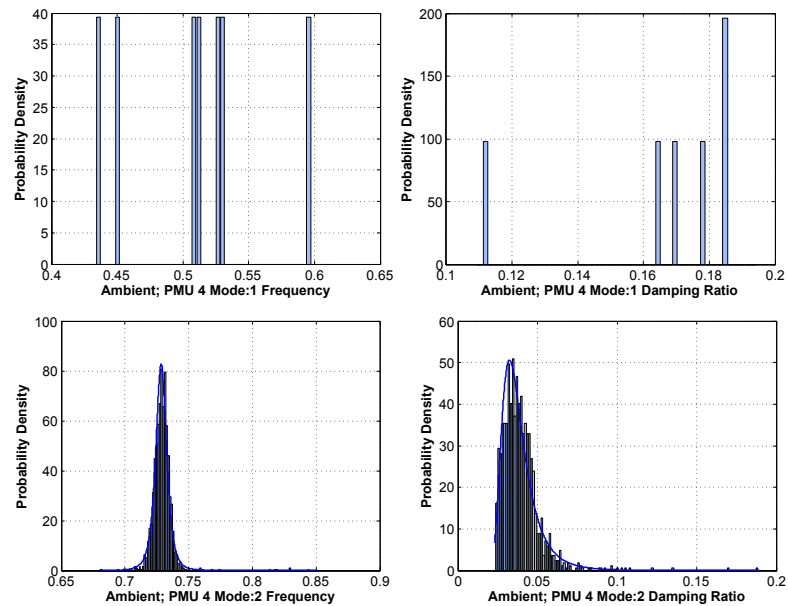

Fig. 2. Estimates form PMU4 data in ambient conditions; TOP: PDF of Mode 1 frequency (left), PDF of Mode 1 damping ratio (right); BOTTOM: PDF of Mode 2 frequency (left), PDF of Mode 2 damping ratio (right).
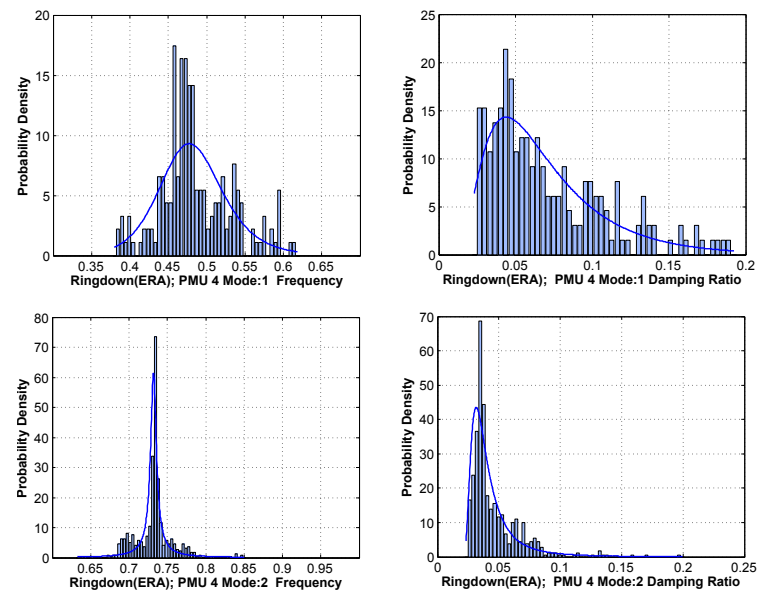

Fig. 4. Estimates form PMU4 data in ringdown conditions; TOP: PDF of Mode 1 frequency (left), PDF of Mode 1 damping ratio (right); BOTTOM: PDF of Mode 2 frequency (left), PDF of Mode 2 damming ratio (right).

bus 49 is quite low when compared to the activity of Mode 2 at the same location. Similar plots could be presented for other PMUs (PMU 2 at bus 40 and PMU 3 at bus 44). As

TABLE II

ANALYSIS OF DECENTRALIZED MODE ESTIMATES FOR AMBIENT DATA

\begin{tabular}{ccccccccc}
\hline & \multicolumn{4}{c}{ Mode 1 } & \multicolumn{4}{c}{ Mode 2 } \\
\cline { 2 - 9 } PMU ID & \multicolumn{2}{c}{ Freq. (Hz) } & \multicolumn{2}{c}{ Damp. (\%) } & \multicolumn{2}{c}{ Freq. (Hz) } & \multicolumn{2}{c}{ Damp. (\%) } \\
\cline { 2 - 9 } & $\mu^{a}$ & $\sigma^{b}$ & $\mu$ & $\sigma$ & $\mu$ & $\sigma$ & $\mu$ & $\sigma$ \\
\hline PMU 1 & .502 & .008 & 5.0 & 1.9 & .732 & .015 & 5.3 & 1.8 \\
PMU 2 & .504 & .01 & 5.1 & 2.0 & .734 & .014 & 5.5 & 1.8 \\
PMU 3 & .512 & .015 & 6.2 & 3.3 & .739 & .017 & 6.1 & 2.5 \\
PMU 4 & .512 & .058 & 16.2 & 3.9 & .729 & .010 & 3.9 & 1.3 \\
\hline True Value & .499 & & 3.18 & & .732 & & 3.52 &
\end{tabular}

${ }^{a} \mu$ : mean

${ }^{b} \sigma:$ Standard deviation 
confirmed by Table II, the signal at PMU 4 will have frequency estimates with higher variance and lower accuracy for Mode 1 as compared with the estimates of Mode 2. The same behaviour can be observed in the mode damping estimates, higher variance and low accuracy for Mode 1 as compared to Mode 2 [9].

2) Ringdown Data: In case of a ringdown, a ringdown condition was identified by the mode-estimator by comparing the oscillation energy of the system's response w.r.t. a fixed threshold. Each time, after the detection of ringdown in form of rising oscillation energy, 200 subsequent samples (representing an impulse response) were transferred to the ERA based ringdown analyzer to obtain a set of mode estimates. The process continued till 6000 estimates were achieved.

The PDF plots of frequency and damping ratio estimates by PMU 1 and PMU 4 for ringdown data are shown in Figs. 3 and 4. Table III presents the mean and standard deviation of the two modes identified in case of ringdown data for all the PMUs which were used in the decentralized architecture of mode estimation. From the Figs. 3 and 4, it can be observed that similar to the PDF plots of modal frequencies in case of ambient data, the activity of Mode 1 observed by PMU 4 at bus 49 is lower than the activity of Mode 2 at the same location. However, because of the impulses present in the response, the visibility of Mode 1 from PMU 4 is higher than the one observed in Fig. 2. This is reflected in the better accuracy and lower variance computed from PMU 4 as shown in Table III. PMU 4 in Table III gives a mean of $0.485 \mathrm{~Hz}$ with standard deviation of 0.051 in comparison of a mean $0.512 \mathrm{~Hz}$ with standard deviation of 0.058 given by PMU 4 in Table II.

TABLE III

ANALYSIS OF DECENTRALIZED MODE ESTIMATES FOR RINGDOWN DATA

\begin{tabular}{ccccccccc}
\hline & \multicolumn{4}{c}{ Mode 1 } & \multicolumn{4}{c}{ Mode 2 } \\
\cline { 2 - 9 } PMU ID & \multicolumn{3}{c}{ Freq. (Hz) } & \multicolumn{2}{c}{ Damp. (\%) } & \multicolumn{2}{c}{ Freq. (Hz) } & \multicolumn{2}{c}{ Damp. (\%) } \\
\cline { 2 - 10 } & $\mu$ & $\sigma$ & $\mu$ & $\sigma$ & $\mu$ & $\sigma$ & $\mu$ & $\sigma$ \\
\hline PMU 1 & .501 & .012 & 4.5 & 1.9 & .737 & .024 & 4.4 & 1.9 \\
PMU 2 & .497 & .011 & 5.0 & 2.1 & .728 & .031 & 4.2 & 1.8 \\
PMU 3 & .496 & .014 & 5.1 & 2.1 & .738 & .039 & 4.7 & 2.4 \\
PMU 4 & .485 & .051 & 7.0 & 4.0 & .729 & .038 & 4.5 & 2.1 \\
\hline True Value & .499 & & 3.18 & & .732 & & 3.52 & \\
\hline
\end{tabular}

3) Centralized Estimation: When the same data obtained from ambient and ringdown conditions measured at all PMU locations were processed by a central estimator, the modes for the whole system were computed without taking advantage of any location specific information. Statistical results obtained by the centralized mode estimator are presented in Table IV.

Note that because these inter-area modes were observable at the the measurement locations, the centralized estimator provides good accuracy and low variance, as shown in Table IV.

\section{B. Observation of Local Forced Oscialltions}

The benefits of using a decentralized mode estimator to monitor modal information on a local level was evaluated in
TABLE IV

ANALYSIS OF CENTRALIZED MODE ESTIMATES FOR AMBIENT AND RINGDOWN DATA

\begin{tabular}{ccccccccc}
\hline & \multicolumn{4}{c}{ Mode 1 } & \multicolumn{4}{c}{ Mode 2 } \\
\cline { 2 - 9 } Condition & \multicolumn{1}{c}{ Freq. (Hz) } & \multicolumn{2}{c}{ Damp. (\%) } & \multicolumn{2}{c}{ Freq. (Hz) } & \multicolumn{2}{c}{ Damp. (\%) } \\
\cline { 2 - 9 } & $\mu$ & $\sigma$ & $\mu$ & $\sigma$ & $\mu$ & $\sigma$ & $\mu$ & $\sigma$ \\
\hline \multirow{2}{*}{ Ambient } & .502 & .009 & 4.6 & 1.9 & .733 & .014 & 5.2 & 1.7 \\
Ringdown & .498 & .016 & 3.6 & 1.2 & .728 & .023 & 4.3 & 2.0 \\
\hline
\end{tabular}

another test trying to observe and estimate a local, forced oscillation. A recurring forced oscillation was superimposed on the random load variation input at bus number 52 with a frequency of $1.75 \mathrm{~Hz}$. Three different amplitudes; 0.4, 0.7 and 1.0 p.u. were tested for amibent data case. Ringdown condition was tested with a forced oscillation of amplitude 7 p.u.. These oscillations were imposed every second. The properties of the forced oscillation (Mode 3 from here onwards) are shown in Table V.

TABLE V

PARAMETERS OF FORCED OSCILLATION

\begin{tabular}{|c|c|c|}
\hline Mode & Frequency & Damping Ratio \\
\hline Mode 3 & $1.75 \mathrm{~Hz}$ & $5 \%$ \\
\hline
\end{tabular}

To observe the three modes, 4 PMUs were placed, one each at bus 38, 40, 44 and 52 . The modes were estimated using both centralized and decentralized architectures. The first test was carried out using ambient data. The results for Mode 3 using both the architectures in the presence of ambient data and different oscillation amplitudes are presented in Table VI. The PDF plots for the estimates in presence oscillations of amplitude 0.7 p.u. are presented in Fig. 5.

TABLE VI

OBSERVATION OF LOCAL MODE 3 USING CENTRALIZED AND DECENTRALIZED ARCHITECTURE FOR AMBIENT DATA

\begin{tabular}{cccccc}
\hline \multirow{3}{*}{ Amplitude (p.u.) } & \multicolumn{5}{c}{ Mode 3 } \\
\cline { 2 - 6 } & \multirow{2}{*}{ Method } & \multicolumn{2}{c}{ Freq. (Hz) } & \multicolumn{2}{c}{ Damp. (\%) } \\
\cline { 2 - 6 } & & $\mu$ & $\sigma$ & $\mu$ & $\sigma$ \\
\hline \multirow{2}{*}{0.4} & Centralized & 1.744 & .068 & 4.94 & 4.22 \\
& Decentralized (PMU 4) & 1.749 & .051 & 3.78 & 3.7 \\
\hline \multirow{2}{*}{0.7} & Centralized & 1.738 & .068 & 4.9 & 3.87 \\
& Decentralized (PMU 4) & 1.751 & .036 & 2.8 & 3.1 \\
\hline \multirow{2}{*}{1.0} & Centralized & 1.74 & .065 & 4.9 & 3.91 \\
& Decentralized (PMU 4) & 1.751 & .021 & 1.74 & 2.59 \\
\hline
\end{tabular}

A similar test was also performed by generating ringdown data through impulse inputs to the state-space model. The PDF plots for the frequency and damping ratio distribution for ringdown data are shown in Fig. 6. The statistical results for Mode 3 using both architectures in the presence of ringdown data is presented in Table VII.

From the results shown in Tables VI and VII and Figs. 5 and 6 , it is clear that the variance for the estimates around Mode 3 is comparatively less when computed individually 

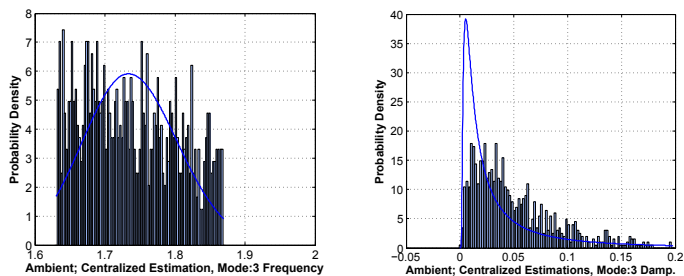

(a)
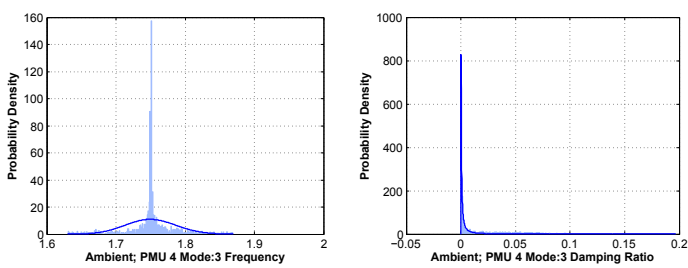

(b)

Fig. 5. PDF for forced oscillation in ambient conditions; (a): centralized estimation, (b): From PMU 4 data in decentralized estimation.
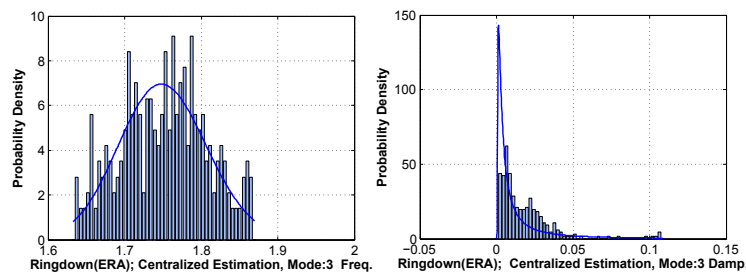

(a)
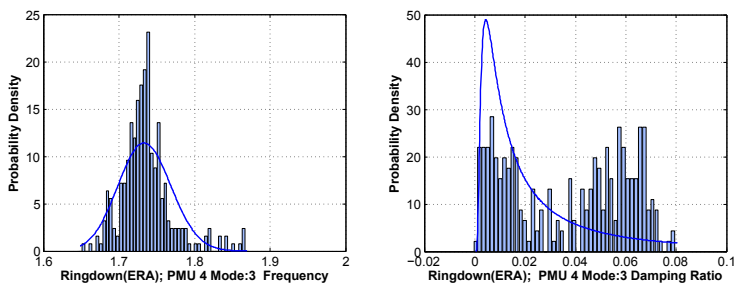

(b)

Fig. 6. PDF for the forced oscillation in ringdown conditions; (a): centralized estimation, (b): From PMU 4 data in decentralized estimation.

TABLE VII

OBSERVATION OF LOCAL MODE 3 USING CENTRALIZED AND DECENTRALIZED ARCHITECTURE FOR RINGDOWN DATA

\begin{tabular}{ccccc}
\hline \multirow{2}{*}{ Method } & \multicolumn{4}{c}{ Mode 3 } \\
\cline { 2 - 5 } & \multicolumn{2}{c}{ Freq. (Hz) } & Damp. (\%) \\
\cline { 2 - 5 } & $\mu$ & $\sigma$ & $\mu$ & $\sigma$ \\
\hline Centralized & 1.751 & .057 & 1.72 & 1.85 \\
Decentralized (PMU 4) & 1.734 & .035 & 3.62 & 2.39 \\
\hline
\end{tabular}

based on data acquired from PMU 4 present at bus 52 . The variance of frequency and damping estimates is significantly lower for ambient data using the decentralized architecture. For ringdown, the variance of frequency is also lower in case of decentralized architecture. Table VI also shows that with increase in the amplitude of forced oscillation, its visibility increases even in centralized estimation but it important to detect the oscillations early and locally which can be achieved by decentralized estimation.

\section{CONCLUSION}

This paper presented test cases and results of utilizing a decentralized architecture for mode estimation. The tests were conducted 'in silico' on KTH-NORDIC 32 network. In the decentralized architecture, each PMU feeds a separate modeestimation process. The results showed that the estimators for each PMU were able to identify the modes present in according to the time-series data received by them. The results proved that decentralized architecture provides a more local and high-resolution awareness in mode observation than the centralized estimation process. It was also shown that this advantage could be utilized in detection of local oscillations. These results provide meaningful insights on the selection of PMU signals for mode estimation, particularly in the presence of local oscillations [10].

Decentralized mode estimation architecture in a more advanced form could be employed by the TSOs to develop a double layered and modular oscillation monitoring and data exchange framework which could be employed seamlessly between multiple local electricity utilities.

\section{ACKNOWLEDGMENT}

This work is supported by the FP7 IDE4L project funded by the European Commission, the STandUp for Energy Collaboration Initiative, Statnett SF (the Norwegian TSO) and by the StrongGrid project funded by the Nordic Energy Research.

\section{REFERENCES}

[1] J.F. Hauer, D.J. Trudnowski, J.G. and DeSteese, "A Perspective on WAMS Analysis Tools for Tracking of Oscillatory Dynamics," in Proc. IEEE Power Engineering Society General Meeting 2007, June 2007, pp. $1-10$.

[2] M. Baudette, V.S. Peric, R.S. Singh, S. Løvlund and L. Vanfretti, "Testing of a Real-Time PMU-Based Tool for Power System Mode Estimation" submitted to Power and Energy Society General Meeting, 2016 IEEE, pp. 1-4.

[3] J. Ning, S.A.N. Samadi, V. Venkatasubramanian, "Two-Level Ambient Oscillation Modal Estimation From Synchrophasor Measurements", in IEEE Trans. Power Systems, vol. 30, No. 6, pp. 2913-2922, Aug. 2015.

[4] Identification of Electromechanical Modes in Power Systems, IEEE Task Force Report, Jun. 2012.

[5] J.W. Pierre, D.J. Trudnowski, M.K. Donnelly, "Initial Results in Electromechanical Mode Identification from Ambient Data”, in IEEE Trans. on Power Systems, vol. 12, No. 3, pp. 1245-1251, Aug. 1997.

[6] J.W. Pierre, D.J. Trudnowski, M.K. Donnelly, "Use of ARMA Block Processing for Estimating Stationary Low-Frequency Electromechanical Modes of Power Systems", in IEEE Trans. on Power Systems, vol. 18, No. 1, pp. 167-173, Feb. 2003.

[7] J. F. Hauer, F. Vakili, "An Ascillation Detector Used in BPA Power System Disturbance Monitor", in IEEE Trans. on Power Systems, vol 5, No. 1, pp. 74-79, Feb. 1990.

[8] Y. Chompoobutrgool and L. Vanfretti, "Identification of Power System Dominant Inter-Area Oscillation Paths", in IEEE Trans. on Power Systems, vol. 28, No. 3, pp. 2798-2807, Aug. 2013.

[9] V. S. Peric, X. Bombois, and L. Vanfretti, "Optimal Signal Selection for Power System Ambient Mode Estimation Using a Prediction Error Criterion", in IEEE Trans. on Power Systems, vol. PP, No. 99, pp. 1-13, Sep. 2015.

[10] L. Vanfretti, S. Bengtsson, V. Peric and J. O Gjerde, "Effects of forced oscillations in power system damping estimation" in International Workshop on Applied Measurements in Power Systems, pp. 1-6, Sep. 2012. 